\title{
PHARMACEUTICAL CARE FOR PATIENTS WITH POSTOPERATIVE HISTORY
}

\author{
Nikolina Zarkova ${ }^{1}$, Anna Todorova ${ }^{2}$, Ivo Kumanov ${ }^{2}$ \\ ${ }^{1}$ Dilyana Pharmacy, Mladost Trade Complex, Varna \\ ${ }^{2}$ Department of Pharmaceutical Sciences and Pharmaceutical Management, \\ Faculty of Pharmacy, Medical University of Varna
}

\begin{abstract}
Surgical wounds are the most commonly treated wounds in today's global medical practice. Often, patients are not aware of exactly how they should treat their post-surgical wounds at home. A model for pharmaceutical care of patients with postoperative wounds was developed on the basis of literary sources and guidelines. The Life Value (LV) of the patients was evaluated using the generic SF12 v2 instrument. SF12 v2 was administered to consulted patients and not consulted patients in a control group. The results showed an improvement in all measured LV scores within the group of the consulted patients. Pharmacists are the most accessible healthcare professionals and with their knowledge and competencies, they can contribute to the process.
\end{abstract}

Keywords: postoperative wounds, pharmaceutical care, quality of life

\section{INTRODUCTION}

In modern health care systems, the demands towards pharmacists (the most accessible medical professionals and a source of health information) are constantly rising. Pharmacy practice is increasingly focusing on patient consultation and patient care (1).

Of all the types of wounds, the most common and prevalent in the world are those from surgical interventions. The results of a study of the prevalence of the different types of wounds show that the most common wounds in the world (112.4 million) are post-operative. Their share exceeds many times all the others, followed by skin ulcers - 37000000 , and diabetic ulcers 14500000 (2). Most of the surgical

Address for correspondence:

Anna Todorova

Department of Pharmaceutical Science

and Pharmaceutical Management

Faculty of Pharmacy

Medical University of Varna

e-mail:anna.todorova@mu-varna.bg

Received: June 7, 2018

Accepted: November 5, 2018 wounds are categorized as acute conditions that are treated without complications for an indefinite period of time (3). Patients are often unaware of the exact way they have to treat their wounds at home. The healing process can be influenced by external and internal factors, resulting in complications and infections that slow the wound healing (4).

Inappropriate wound healing leads to additional treatment costs associated with the need for re-hospitalization, additional medicinal products and consumables, additional surgery, and in some cases may turn into a life-threatening condition (5). The large proportion of patients with post-operative wounds and the existing risks of complications determine the social significance of the problem and require the need for effective collaboration among all health professionals (doctors, pharmacists, nurses, etc.).

As the most accessible healthcare professionals who have gained patients' trust (6), pharmacists ought to have the necessary knowledge and competencies related to all phases of the wound healing process, innovative medical devices and appropriate dressings that facilitate the healing process and re- 
duce the risk of developing an infection. The knowledge of wound healing and the opportunities to influence this dynamic and complicated process have increased significantly over the past few years (7). Regardless of the type of the wound and the degree of tissue loss, the healing of each wound takes place in phases that overlap in time and cannot be separated from one another (8). The international guides for wound healing are as follows: NICE, T.I.M.E., and Consensus Document of the World Union of Wound Healing Societies. In Bulgaria, no single consensus has yet been reached on the development of guidance to help medical professionals (9-11). T.I.M.E. is a practical tool adapted to the phases of the wound process, assisting practitioners when assessing and making decisions for the treatment of patients with wounds. Each letter of the abbreviation corresponds to a particular process that is essential for the treatment and progression of the wound process as follows: $\mathrm{T}$ (tissue management), I (inflammation and infection control), $\mathrm{M}$ (moisture balance), and $\mathrm{E}$ (epithelial/edge/advancement) (12).

The wide variety of innovative wound medical devices on the market requires specific expertise from medical professionals related to the proper treatment and choice of appropriate dressing for each of the phases of the healing process. Based on the condition of the issue, we have developed a model for pharmaceutical care (PC) for consulting patients with post-operative wounds and to investigate the role of the pharmacist in optimizing the recovery of patients undergoing specialized PCs.

\section{MATERIALS AND METHODS}

A documentary analysis was carried out with a reference to the available literature sources for wound healing - NICE, T.I.M.E., a consensus paper from the World Union of Wound Healing Societies. A model for PC, adapted for use by practicing pharmacists based on the T.I.M.E. guideline, was developed to best fulfill the needs of pharmaceutical practice in our country.

The study was conducted in pharmacies on the territory of Varna with the cooperation of the Orthopedic Department of the St. Anna University Hospital, Varna. It covers the period of June to September, 2017.
Object and scope of the study were patients with postoperative wounds who had had a surgical intervention in the Orthopedic Department of the St. Anna University Hospital, Varna and were counseled by a pharmacist at a pharmacy following the steps of the developed algorithm, and a control group of patients who did not receive pharmaceutical care at a pharmacy, according to the model. Criteria for inclusion were: patients in the Orthopedic Department of the St. Anna University Hospital with post-operative wounds irrespective of the location of the wound, the age of the patient, and the presence of a concomitant disease. Exclusion criteria included patients who refused to participate in the study and patients who could not look after themselves and could not move alone.

The effectiveness of the PCs was evaluated by assessing the Life Value (LV) of the two patients groups. Patients' quality of life was measured by using the SF12 v2 generic tool (short form of SF 36). SF12 V2 Health Survey is a validated Bulgarian questionnaire. It consists of 12 questions for assessing physical and mental health within the last 4 weeks. The results were measured with a software program provided by Optum, the product license holder. The participants responded to the questions from the paper test handed to them after giving an unequivocal consent and receiving instructions on how to fill their information in.

\section{RESULTS}

The developed PC model is adapted for use by actively working pharmacists on the basis of the T.I.M.E. guideline, adequately in line with the needs of the pharmaceutical practice in our country. The proposed algorithm mainly aims at choosing a therapeutic approach and appropriate dressing depending on the type and condition of the wound, and in case of suspicion of complications, the patients are immediately referred to a physician consultation (Fig. 1).

The study involved a total of 58 people in a postoperative recovery period with surgical wounds in different locations. Thirty-eight patients were consulted on the proposed pharmaceutical care scheme, and 20 were the control group of unconsulted patients. The SF-12 v2 questionnaire is based on the Quality Metric 2009 Norming Study conducted in the United States in 2009 by the National Research 


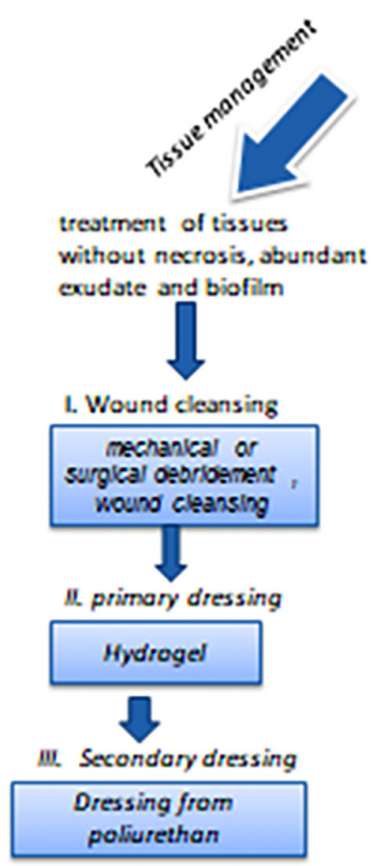

without necrosis, obundant

exudste and biofilm

1. Wound cleansing

chanical or

cal oeprloament wound cleansing

II primary dressing

Secondary dressing

poliurethan

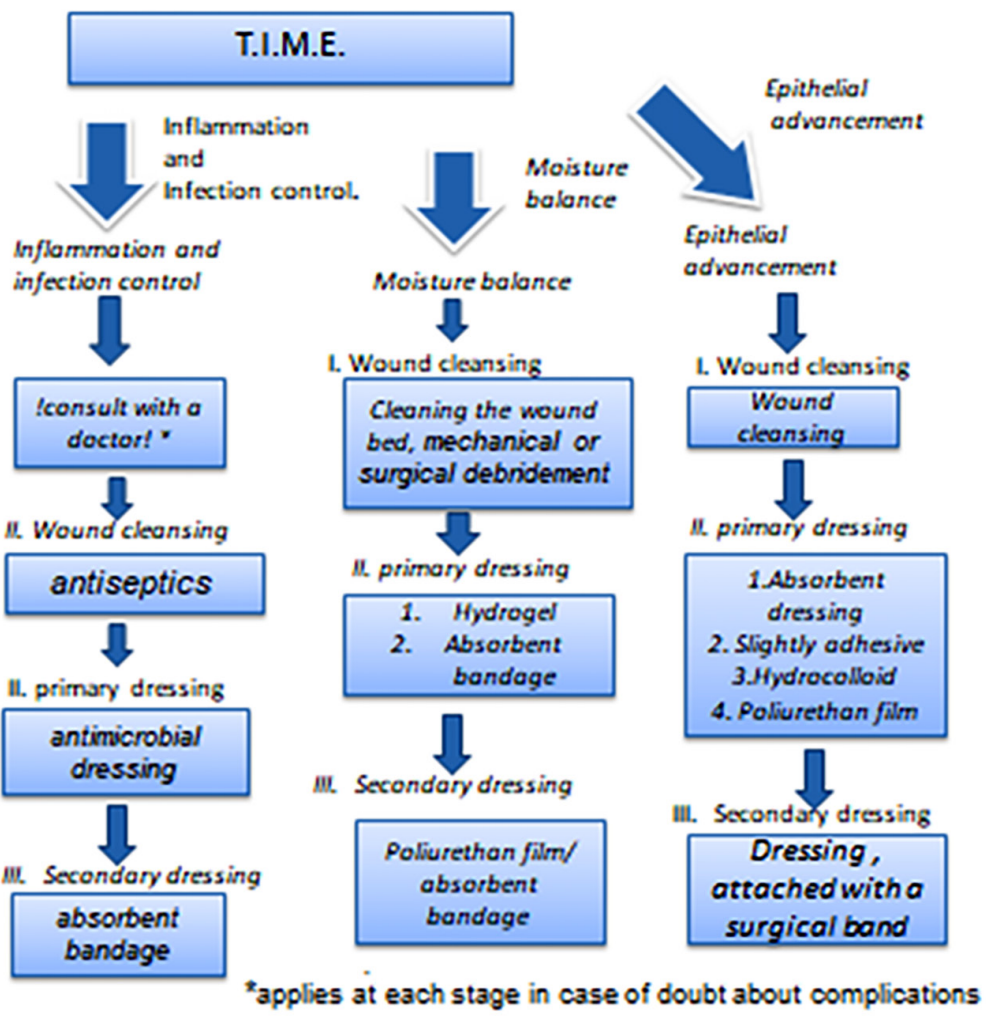

Fig. 1. Algorithm for therapeutic approach and choice of appropriate dressing

Corporation (NRC) based on which the scale used for the study was determined as well as the norm (50 points) for the investigated health indices with a minimum value of 20 and a maximum value of 80 . Higher scores on the scale are an indicator of better quality of life.

Results of the quality of life tests of the consulted and unconsulted patients with post-operative wounds are presented below.

In the group of pharmacist-consulted patients subject to specialized PC, it appeared that the mental component summary (MCS) exceeded the established norm. The individual components of mental health that made up the indicator were above or matched exactly the norm. An exception was the emotional component RE, which could be explained by the recent intervention. Unlike MCS, the physical component summary (PCC) showed values below the norm, with the highest general health $(\mathrm{GH})$ component and the rest having approximately similar values (Fig. 2).

Figure 3 summarizes the physical and mental health assessments and the individual components,

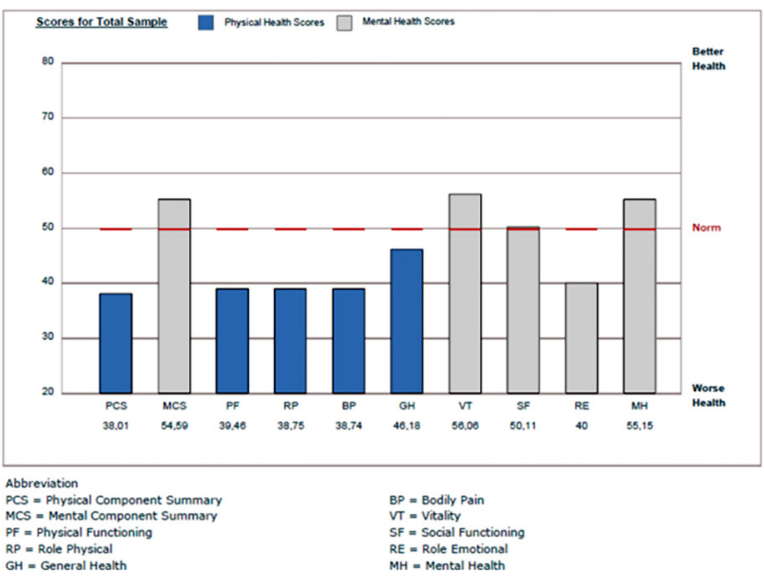

Fig. 2. Results of mental and physical health tests in consulted patients with postoperative wounds

which were used in the quality of life test conducted in non-consulted patients.

The results for non-consulted patients were below the norm for the individual indicators. Postoperative patients with wounds, not advised by pharmacists, have been found to have reduced general physical functioning as well as reduced overall mental health indicators. 


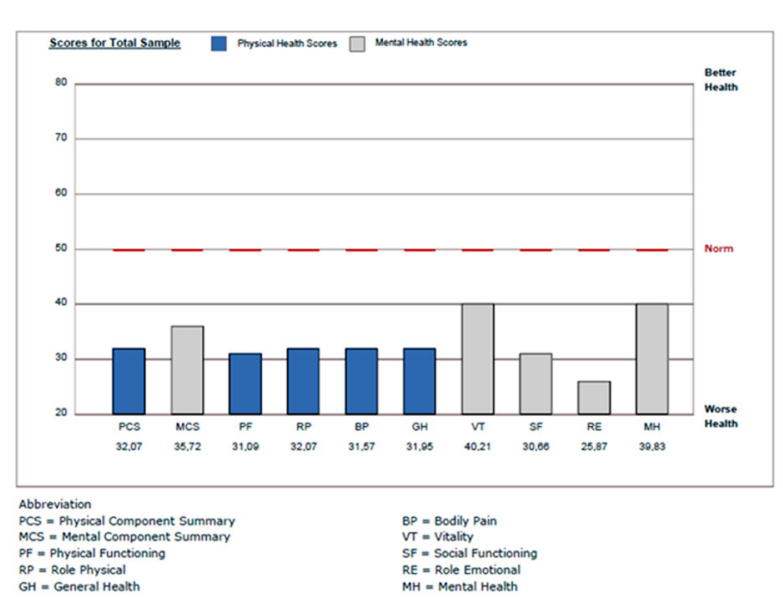

Fig. 3. Results of a physical and mental health test conducted on a non-consulted patients

The study results make it possible to detect the risk of developing depression in the study population at a total population rate of $20 \%$.

The results obtained showed that $90 \%$ of patients not advised by a pharmacist were at risk of developing depression.

Patients under PC, who were consulted at a pharmacy, had very different results, with the risk of depression being only $2 \%$. The distribution of patients with a risk of developing depression from the two study groups - consulted and non-consulted control group is presented in Figures 4 and 5 .

Figure 6 and Figure 7 summarize the results for the assessment of physical (PCS) and mental (MCS)

\section{First Stage Positive Depression Screening: $\%$ at Risk}

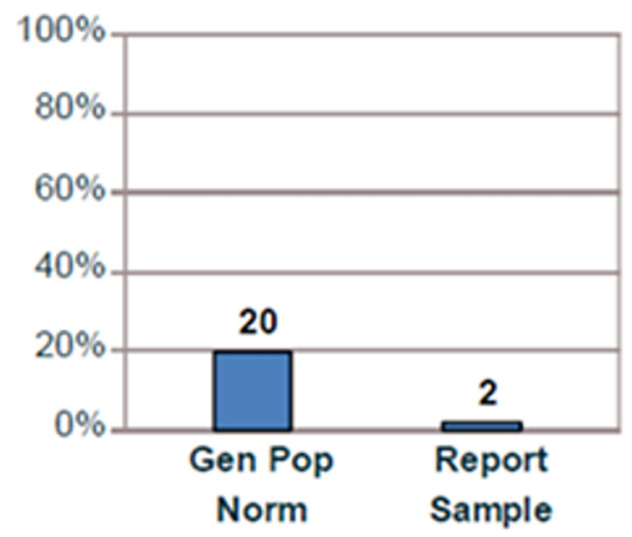

Fig. 4. Risk of depression in consulted patients

\section{First Stage Positive Depression}

\section{Screening: $\%$ at Risk}

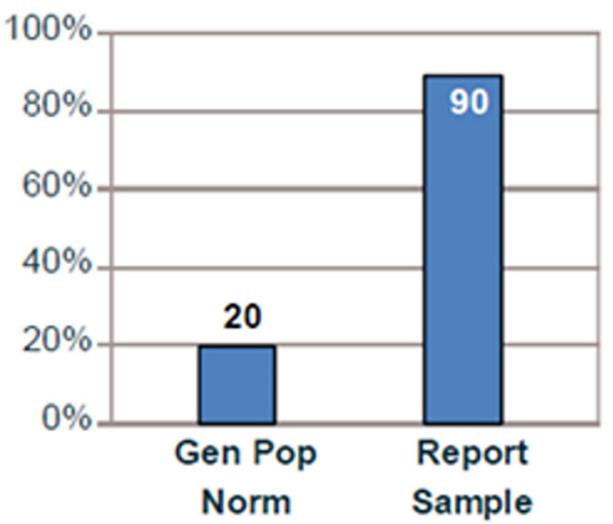

Fig. 5. Risk of depression in unconsulted patients

\section{\% Sample whose Scores are Above, At or Below the General Population Norm}

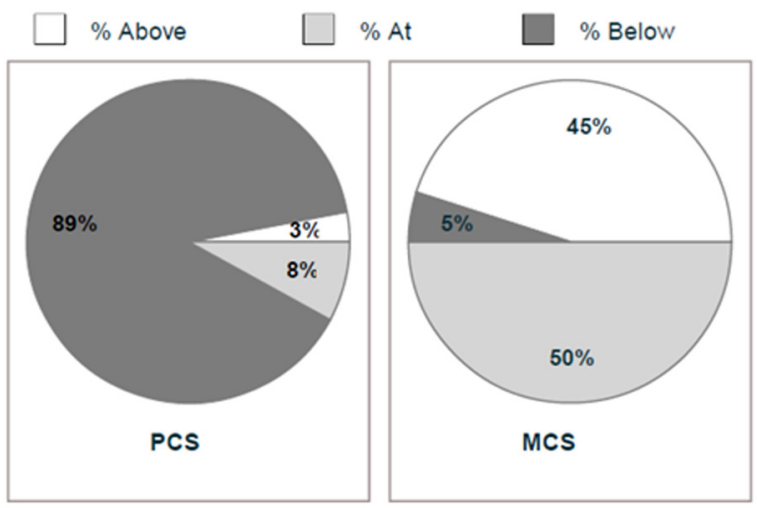

Fig. 6. Physical and psychological status of consulted patients

health in the study groups of patients compared to the generally established norm.

Reduced results were found for non-consulted patients, and for MCS and PCS. As shown in Figure 7 , in terms of physical health, $100 \%$ of the patients included in the study were below the general norm and, in regard to mental health, $95 \%$ were below the established norm.

In the case of the consulted patients it was found that $89 \%$ of the studied individuals had physical health below the norm. Unlike the non-consulted, where $8 \%$ of the patients were within the norm for physical health and 3\% were above the norm. Figure 6 shows that, in terms of mental health, $50 \%$ of respondents were within the norm, $45 \%$ were above 


\section{$\%$ Sample whose Scores are Above, At or Below the General Population Norm}

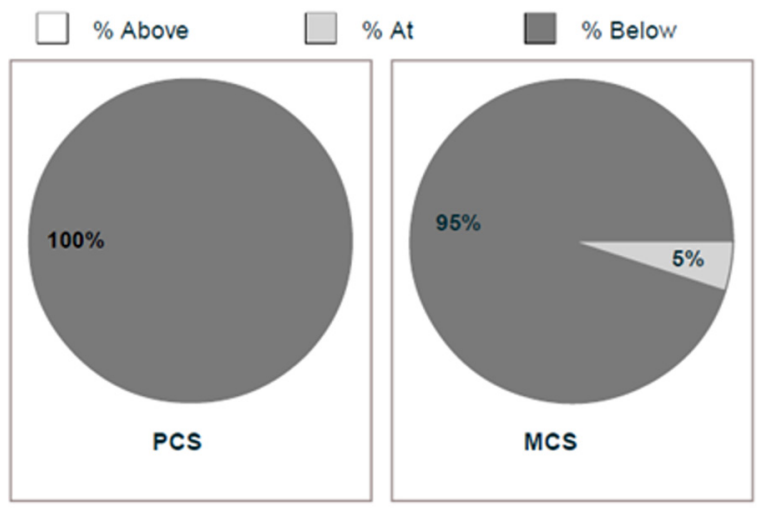

Note: Totals may not equal $100 \%$, due to statistical rounding.

Fig. 7. Physical and psychological status of unconsulted patients

the norm, and only $5 \%$ were below the norm for mental health.

For better illustration and evaluation of the indicators in the two groups of patients - consulted and non-consulted by a pharmacist, the results are summarized and compared in Figure 8.

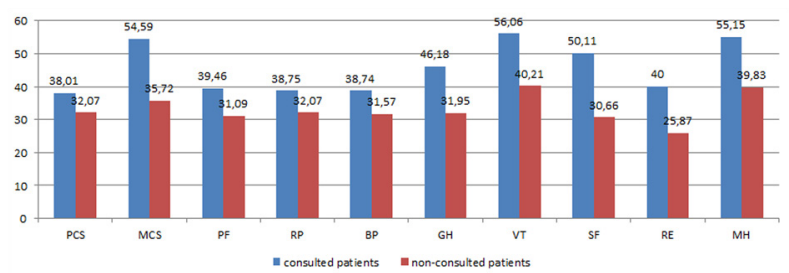

Fig. 8. Comparative analysis of mental and physical health of consulted and non-consulted patients with postoperative wounds

The figure shows that the mental health level of the patients consulted was high and in some categories (VT, MH, SF), even above the universally established norm. The difference in overall physical health indicators for both groups of patients was approximately 6 points in favor of those consulted, although both groups remained below the norm.

It is noteworthy that all the parameters of consulted patients were better than those obtained in non-consulted patients.

\section{DISCUSSION}

The introduction of the concept of Pharmaceutical Care in Pharmacy Practice places emphasis on the observation of the results of the applied treatment (13). With self-medication with over-the-counter (OTC) medicines, medical devices and more, much of the responsibility for patient health has been shifted to the pharmacists and their care (14).

Unconsulted patients with postoperative wounds showed poor quality of life on all tested parameters - general physical, mental health and social functions. The reduced result for PCS in both groups of patients is considered acceptable, based on their condition due to the recent surgery and the currently impaired health. It should be noted that in spite of the lower PCS values in the patients with the highest score, GH level is assumed to be a result of specialized PC.

The quality of life of pharmacist-consulted patients exceeded the average mental health indicator and showed improvement in individual components. An exception was RE, which we explain with the stress and discomfort of the recent interventions. Results from other studies have shown that specialists can improve the quality of life of patients $(15,16)$.

Postoperative patients with wounds, who had not been consulted by pharmacists, had significantly impaired overall mental health in all components. For them, the risk of developing depression was extremely high, and low compliance and unsatisfactory end result of treatment could be expected. Poor compliance can lead to a number of complications that may further aggravate the condition and increase the patient's treatment process.

The application of PC in pharmacies can not only improve the quality of life of patients, it may also prevent further complications, such as illnesses, as well as provide efficient and cost-effective treatment $(17,18)$.

For the training of patients and improved compliance, pharmacists should have the necessary knowledge and skills to know the appropriate medical devices and dressings for each stage of the wound process, to recognize the symptoms of a developing wound complication so they can properly advise patients or refer them to a specialist physician when necessary.

\section{CONCLUSION}

Specialized PC in patients with post-operative wounds can contribute to the healing process in re- 
gard to proper wound healing, rapid recovery and avoidance of complications, and hence improve the quality of life of patients after surgery.

Considering the social significance of the problem related to the treatment of post-operative wounds and the results for the improvement of the patients in specialized pharmaceutical care in QoL, it is necessary to create a basic guideline for our country, to help the medical specialists in the treatment and consultation of patients with wounds. Collaboration between all specialists involved in the care of patients with postoperative wounds is also needed for improving the process of healing of patients with postoperative wounds.

\section{REFERENCES}

1. Todorova A. Pharmaceutical Care. Modern pharmaceutical approaches in uncontrolled patients with allergic rhinitis. Medical University Varna; 2016. p. 124. ISBN 978-619-7137-97-2 (in Bulgarian).

2. Kalorama Information, Advanced Wound Care Markets Worldwide, 2012. Available at: http://www.kaloramainformation.com/ Wound-Care-Skin-7032918/

3. Dabiri G, Damstetter E, Phillips T. Choosing a wound dressing based on common wound characteristics. Adv Wound Care. 2016; 5(1): 32-41.

4. Baxter $\mathrm{H}$ Management of surgical wounds. Nursing Times. 2003;99(13): 66-8.

5. Surgical site infections: prevention and treatment Clinical guideline [CG74] Published date: October 2008 Last updated: February 2017 Available at: https://www.nice.org.uk/ Guidance/CG74

6. Pharmaceutical Group of European Union 2012. Available at: www.pgeu.eu

7. Milne J, Vowden P, Fumarola S, Leaper D. Postoperative incision management made easy. Wounds UK. 2012;8(4):1-4, Available from www.woundsuk.com/made-easy

8. Bratzler DW, Hunt DR. The surgical infection prevention and surgical care improvement projects: national initiatives to improve outcomes for $\mathrm{pa}$ tients having surgery. Clin Infect Dis. 2006; 43(3): 322-30.

9. https://www.nice.org.uk/guidance/

10. Dowsett $\mathrm{C}$, Newton $\mathrm{H}$. Wound bed preparation: TIME in practice. Wounds UK. 2004; 12:58-70.
11. Wellbeing and concordance. Minimising pain at wound dressing-related procedures: a WUWHS consensus document,14 October 2009. Available at: https://www.woundsinternational.com/resources/ details/minimising-pain-wound-dressing-relatedprocedures-wuwhs-consensus-document

12. Scott S., Razzano L., Wong E.M. Reducing readmissions related to pressured ulcers Available at: http://www.ppahs.org/wp-content/uploads/2016/12/PPAHS Readmissions-Pressure-Ulcer-Prevention.pdf

13. Petkova V, Andreevska K, Gueurguiev S, Grekova D, Dimitrov M, Voycheva C, et al. Community pharmacy-based program for patients with asthma and chronic obstructive pulmonary disease. Biotechnol Biotechnol Equip. 2012; 26 (6) : 3437-42.

14. Tsvetkova A, Todorova A, Petkova V. The role of pharmacist as an advisor on responsible selfmedication: a survey. World J Pharm Pharm Sci. 2014;3(11): 325-35. Available at: https://www.researchgate.net/publication/267631564_THE_ ROLE_OF_PHARMACIST_AS_AN_ADVISOR _ ON_RESPONSIBLE_SELF-MEDICATION_A_ SURVEY

15. Todorova A, Tsvetkova A, Mihaylova S, Andreevska K, Kondova A, Arnaoudova M. The impact of pharmaceutical care on improving the quality of life in patients with allergic rhinitis. CBU International Conference Proceedings. 2017; 5:1022-7. Available at: https://www.researchgate.net/publication/320497583_THE_IMPACT_OF_PHARMACEUTICAL_CARE_ON_IMPROVING_THE_ QUALITY_OF_LIFE_IN_PATIENTS_WITH_ALLERGIC_RHINITIS

16. Todorova A, Tsvetkova A. Role of pharmaceutical care in treatment of pediatric bronchial asthma. World J Pharm Pharm Sci. 2015; 4(1): 38-51.

17. Kondova A, Todorova A, Tsvetkova A, Arnaoudova M, Andreevska K, Grekova D. Screening and risk assessment for depression in community pharmacy- pilot study. J IMAB. 2018; 24(1):1928-31.

18. Grekova D, Dimitrova Z, Andreevska K, Madjarov V, Georgiev S. Pharmacoeconomic cost-effectiveness analysis of standard care for patients with moderate asthma and pharmaceutical care in pharmacies. Scientific papers of the Union of Scientists in Bulgaria-Plovdiv, Series G. Medicine, Pharmacy and Dental Medicine. 2013; 14:107-12. (in Bulgarian). 